\title{
Role of serial order in the impact of talker variability on short-term memory: testing a perceptual organization-based account
}

\author{
Robert W. Hughes • John E. Marsh • Dylan M. Jones
}

Published online: 3 June 2011

(C) Psychonomic Society, Inc. 2011

\begin{abstract}
In two experiments, we examined the impact of the degree of match between sequential auditory perceptual organization processes and the demands of a short-term memory task (memory for order vs. item information). When a spoken sequence of digits was presented so as to promote its perceptual partitioning into two distinct streams by conveying it in alternating female (F) and male (M) voices (FMFMFMFM) - thereby disturbing the perception of true temporal order - recall of item order was greatly impaired (as compared to recall of item identity). Moreover, an order error type consistent with the formation of voice-based streams was committed more quickly in the alternating-voice condition (Exp. 1). In contrast, when the perceptual organization of the sequence mapped well onto an optimal two-group serial rehearsal strategy - by presenting the two voices in discrete clusters (FFFFMMMM) - order, but not item, recall was enhanced (Exp. 2). The results are consistent with the view that the degree of compatibility between perceptual and deliberate sequencing processes is a key determinant of serial short-term memory performance. Alternative accounts of talker variability effects in short-term memory, based on the concept of a dedicated phonological short-term store and a capacity-limited focus of attention, are also reviewed.
\end{abstract}

Keywords Short term memory · Talker variability · Serial recall $\cdot$ Perceptual organization

The capacity to retain and reproduce the serial order of a sequence of verbal stimuli over the short term has long commanded interest, on the grounds that such a capacity is

R. W. Hughes $(\bowtie) \cdot$ J. E. Marsh • D. M. Jones

School of Psychology, Cardiff University,

Cardiff CF10 3AT, UK

e-mail: HughesRW@cardiff.ac.uk implicated in fundamental activities such as speech comprehension and language acquisition (e.g., Baddeley, Gathercole, \& Papagno, 1998). The present article is concerned with a largely neglected phenomenon whereby serial short-term memory (STM) for spoken sequences is markedly poorer when successive items are spoken in different voices-for instance, alternating female (F) and male (M) voices - as compared to the standard, single-voice method of presentation (e.g., Goldinger, Pisoni, \& Logan, 1991; Greene, 1991; Hughes, Marsh, \& Jones, 2009; Martin, Mullennix, Pisoni, \& Summers, 1989). Recent evidence has suggested that alternating voices incur a cost because the sequence is obligatorily organized perceptually into streams defined by voice, such that there is a conflict between the order of items as perceived and the requirement to reproduce the items according to their true temporal order (Hughes et al., 2009). Here we test a key prediction of this perceptual organizationbased account: Given the same list, a task that demands the retention of item order should be impaired by voice alternation to a greater extent than a task that requires the retention of item information (without respect to order).

The classic test of verbal serial STM involves presenting a list of familiar verbal items in an unfamiliar order (e.g., random permutations of the digits 1-8) and, following the last item, requiring the participant to reproduce either the entire sequence (serial recall-e.g., Conrad, 1964) or part of the sequence in response to a probe (e.g., "which item followed item $x$ ?"; probed order recall-e.g., Murdock, 1968). A standard feature of studies of serial STM for spoken material is that each item in the list is presented in the same voice. Whilst this may be convenient experimentally, the near ubiquity of this methodological feature tends to obscure the possibility that the singularity of voice plays a key role in auditory-verbal serial STM. Indeed, a handful of studies have demonstrated that when successive items are presented 
in different voices, serial recall is impaired appreciably (Goldinger et al., 1991; Greene, 1991; Hughes et al., 2009; Martin et al., 1989; Nygaard, Sommers, \& Pisoni, 1995). For example, when a male and female voice are alternated, serial recall of digit lists is far poorer than when one of the voices delivers the entire list (Greene, 1991; Hughes et al., 2009).

We have recently garnered evidence for the view that the benefit of voice singularity on serial recall is related to auditory perceptual organization processes (Hughes et al., 2009). These refer to the obligatory processes that serve to integrate, or otherwise partition, successive auditory stimuli into streams according to whether or not they share a common origin, based on Gestalt principles such as similarity of pitch and timbre, "good continuation," and so forth (e.g., Bregman, 1990; Macken, Tremblay, Houghton, Nicholls, \& Jones, 2003). For instance, when two tones that are particularly distinct from one another in pitch - and hence unlikely to have originated from the same source - are alternated (e.g., ABABABAB...), the sequence tends to be partitioned perceptually into two interleaved streams defined by pitch (i.e., AAAA and BBBB; Bregman \& Rudnicky, 1975; Miller \& Heise, 1950; Van Noorden, 1975). Importantly, it is well established that the serial order of elements within a stream is relatively easy to discern, but it is notoriously difficult to perceive the order of elements that belong to different streams (e.g., Bregman \& Campbell, 1971; Van Noorden, 1975). In Bregman's (1990) words, "the listener loses the finer details of the temporal relation between sounds that are in different streams and focuses instead on the temporal relations that exist among the elements of the same stream" (p.143).

From this standpoint, serial recall items that are delivered in the same voice, given their shared pitch range, timbre, and so on, are likely to be organized obligatorily into a single coherent stream such that there would be little ambiguity, in perceptual terms, regarding their serial order (Bregman \& Rudnicky, 1975). As such, the perceptual organization of a single-voice list yields order cues that are compatible with the strict serial-order demands of the task. Voice alternation may cause an ambiguity as to the serial order of the items because the tendency for items to be streamed automatically by spectral similarity means that the order of nonadjacent items - those in the same voice - may be more readily perceived than (or as readily perceived as) the order of adjacent items (Bregman \& Rudnicky, 1975). Thus, the perceptual organization of the list in such a condition is incompatible with the requirement to reproduce the items in strict serial order (Hughes et al., 2009). Evidence for the perceptual organization-based account includes the finding that factors known to promote perceptual segregation accentuate the damaging effect of voice alternation: If a (not-to-be-recalled) lead-in of items is presented in one of the voices conveying an ensuing to-be-remembered list of digits presented in alternating voices (e.g., an MMMMM lead-in followed by an MFMFMF to-be-remembered list), the effect is larger than in the absence of the lead-in (Hughes et al., 2009, Exp. 1). This accentuation does not occur if the lead-in is in a different voice from either of those conveying the following list (Hughes et al., 2009, Exp. 2). Such effects are also readily explained by principles of streaming: The lead-in of $\mathrm{M}$ items serves to establish a stable stream into which the same-voice $\mathrm{M}$ items in the subsequent to-be-remembered list can be assimilated, so that the $\mathrm{F}$ items are more readily "thrown out" perceptually into their own separate stream (Anstis \& Saida, 1985; Rogers \& Bregman, 1993).

A further prediction of the perceptual organization-based account tested here is that the voice alternation effect should be evident to the extent that the STM task requires the recall of order information; tasks that only require memory for individual item information should not be so affected by the manner in which the items are ordered by obligatory streaming processes. In the present study, therefore, we vary the degree to which the memory task involves order recall, as well as varying whether the passive perceptual organization of the sequence is at odds with (Exp. 1) or compatible with (Exp. 2) serial-order retention.

To date, there exists only scant and equivocal evidence pertaining to whether varying the talkers conveying a list impairs memory tasks that do not require order recall. For example, Watkins and Watkins (1980) found impaired recall of early list items in a talker-variable condition (in which each item was conveyed in a unique voice) under free recall instructions, whereas Martin, Mullennix, Pisoni, and Summers (1987), cited in Goldinger et al., 1991) found that ordered but not free recall was poorer with multiple-voice presentation. To complicate matters further, despite the fact that, nominally, free recall allows recall of items in any order, participants tend nevertheless to adopt a serial rehearsal strategy in order to perform at least part of the task (Beaman \& Jones, 1998; Bhatarah, Ward, \& Tan, 2008). Thus, the free recall task does not hermetically isolate item from order retention processes, and hence this evidence is moot with regard to whether talker variability affects non-order-based tasks. Moreover, the talkervariable condition in all of these studies involved presenting each item in a unique voice, a procedure that may have implications quite distinct from those flowing from two voices alternating (see the General Discussion). To our knowledge, the present study is the first to directly contrast the impact of voice alternation on order- and item-based memory tasks.

In the present experiments, we used two tasks that were identical in terms of both what was presented and their response-output requirements, but only one task required the retention of serial order (probed order recall; e.g., Murdock, 1968); the other task is thought to require only the retention of item, and not of serial-order, information (missing-item task; e.g., Buschke, 1963; Buschke \& Hinrichs, 1968). In the 
missing-item task, all but one item from an overlearned closed set are presented in a random order (e.g., different random permutations of eight items from the numerals 1-9), and the task on each trial is to identify which item was left off the list. Whereas this task involves memory for each item presented - so as to determine which of the set was not-it does not require the retention of the particular random order in which they occurred (Buschke, 1963; Klapp, Marshburn, \& Lester, 1983). Accounts of missing-item recall thus tend to appeal to processes involved in judgments of individual item familiarity (or, more accurately, unfamiliarity) — which may be supported by a scan of the long-term memory representation of the ordinal sequence (see, e.g., Buschke \& Hinrichs, 1968; Humphreys \& Schwartz, 1971) — rather than to processes relating to order within the presented sequence.

The probed order recall task involves the same presentation conditions and output requirements as the missing-item task (the latter feature making it a better comparison task than serial recall; Beaman \& Jones, 1997), but at test, one item from the just-presented list is re-presented, and the item that followed it in the list must be reported. In this task, therefore, the serial order of the presented items is clearly critical. Independent corroboration for this assumption comes from the fact that probed order recall (like serial recall) is sensitive to disruption by variables thought to impair serial-order processing, such as changing-state irrelevant sounds (e.g., Jones \& Macken, 1993) and articulatory suppression (e.g., Macken \& Jones, 1995), whereas the missing-item task is immune to these variables (Beaman \& Jones, 1997; Hughes, Vachon, \& Jones, 2007; Jones \& Macken, 1993; Klapp et al., 1983; Macken \& Jones, 1995).

\section{Experiment 1}

We begin the series by examining a situation in which the products of perceptual organization should be incompatible with strict serial reproduction and, hence, should result in an impairment of probed order recall, as compared to missing-item recall. Participants were required to undertake both tasks under conditions of single and alternating (female-male) voice presentation. We predicted that voice alternation - a presentation format that tends to promote the perceptual organization of items by voice (Hughes et al., 2009), and hence an organization that is incompatible with the true temporal order of the items - would impair probed order recall as compared to missing-item recall.

\section{Method}

Participants A group of 26 participants from Cardiff University took part, each of whom reported normal or corrected-to-normal vision and normal hearing.
Apparatus and materials The memory lists comprised eight digits taken from the nine-item set of numerals 1-9. Each item was recorded twice, once in a female voice and once in a male voice, and sampled with 16-bit resolution at a sampling rate of $44.1 \mathrm{KHz}$ using Sound Forge 5 (Sonic Inc., Madison, WI). The female and male voices were clearly distinct due to differences in fundamental frequency and timbre, and within each voice the items were spoken at an approximately even pitch. Each item was edited to $250 \mathrm{~ms}$ in duration using the same software. For each list, the digits were presented in a different quasirandom order, with the constraint that no more than two ascending or descending runs of two or more digits (e.g., 2-3 or 7-6) occurred within a given list and that there were no runs of three or more digits. This was also the case for nonadjacent items (e.g., those in Positions 1 and 3), so that in alternating-voice lists there were no more than 2 two-digit runs within a given voice in a given list. The lists were presented at approximately 65-70 $\mathrm{dB}(\mathrm{A})$ over stereo headphones with an interstimulus interval (ISI; offset to onset) of $100 \mathrm{~ms}$. The stimuli were presented using the SuperLab software (Cedrus Corporation).

Design There were two repeated measures factors: List Type (single vs. alternating voice presentation) and Task (missing-item or probed order recall). In one block of trials, the task was to identify and recall the missing item, whereas in the other block, one item (a probe) was presented from the list, and participants were required to recall the item that had followed it in the list. Within each block, there were 18 trials on which all items were presented in the same voice (9 all-female lists and 9 all-male lists: the single condition) and 18 trials on which the lists were presented in an alternating-voice fashion ( 9 starting with a female item, 9 with a male item: the alternating condition). Within each block, no trial type was presented more than twice in succession. For the missing-item task, for each 18 trials within each voice condition, each of the nine possible digits was missing twice. In order to have equal numbers of trials per task per condition (18), for the probed task some positions had to be sampled more frequently than others: Each of the seven Positions 2-8 within a sequence was probed at least twice, but Positions 5 and 6 were probed four times each (to preempt the results, this extra sampling of some positions in the probe task did not affect the pattern of data). Within these constraints, the missing/probed item was chosen randomly for each trial. The order in which the two task-type blocks were undertaken was counterbalanced across participants. There were 2 practice trials (1 single and 1 alternating) preceding each block.

Procedure Participants were tested in groups of up to 4 in a sound-attenuated room, with each participant placed in a 
separate cubicle with its own PC and headphones. For the missing-item block, $50 \mathrm{~ms}$ after the offset of the last item, "which item was missing?" appeared on screen. Participants had $10 \mathrm{~s}$ to indicate - using the number pad on the keyboard - the digit that they thought was missing from the just-presented list. For the probed order recall block, "which item followed $x$ ?" appeared, where $x$ represents one of the digits presented in the just-presented list. Again, participants had $10 \mathrm{~s}$ to respond using the number pad. As soon as a response was made, or after the 10 -s time limit, a 200-ms tone sounded to signal that the first digit of the next list was imminent. The experiment lasted approximately $20 \mathrm{~min}$.

\section{Results and discussion}

Figure 1 shows the percentages of responses in which the missing item (for the missing-item task) or the correct digit given the probe (probed order recall) was identified in each list-type condition. There were main effects of list type, $F$ $(1,25)=55.14, M S E=.009, p<.01$, and task, $F(1,25)=$ $10.14, M S E=.022, p<.005$, and, most importantly, a reliable interaction between list type and task, $F(1,25)=$ $11.40, M S E=.009, p<.005$, reflecting the fact that the impairment due to voice alternation was much larger in the probed order recall task. Simple effects analyses (Fisher's LSD) showed that the missing-item task $(p<.01)$ and the probed order recall $(p<.001)$ task were nevertheless both impaired by voice alternation.

In a supplementary analysis, we checked whether or not the extra sampling of some positions in the probe task had any influence on the results. We omitted the data from trials in the probe task on which a position was being sampled for the third or fourth time, so that each position, as for each missing item, was sampled just twice, and obtained the same pattern of results, with mean correct recall in the probe task being $55.2 \%$

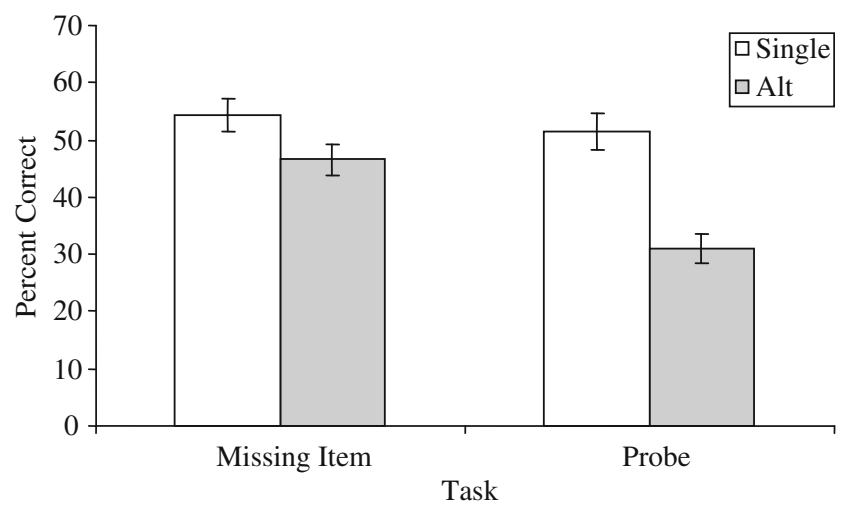

Fig. 1 Mean percentages of items correctly recalled in the single- and alternating-voice conditions in the missing-item task and the probed order recall task in Experiment 1
$(S E=3.44 ;$ single voice $)$ and $33.5 \%(S E=2.67$; alternating voices). It is also worth noting that there was no difference in performance across the two tasks in the baseline (i.e., singlevoice) condition, $p=.45$ ( $p=.81$ using even sampling in the probe task). This helps rule out any interpretation of the differential sensitivity of the two tasks to the impact of voice alternation in terms of differences in task difficulty.

During the peer review process, it was suggested that if, as we suppose, voice-based streaming occurs in the alternating-voice condition and is responsible for the impairment in the probe task, an error type whereby the next-but-one item is output - that is, the item that in the alternating-voice condition was the next item in the same voice in which the probe item was conveyed - should be disproportionately represented among the errors made in that condition, as compared to the single-voice condition. Based on the even-sampling version of the probe task analysis, the proportion of errors accounted for by next-butone-item responses was indeed greater in the alternatingvoice condition $(M=.32, S E=.032)$ than in the singlevoice condition $(M=.24, S E=.037)$, but this difference did not reach significance $(p=.12)$. On reflection, however, it is plausible that this may not be a very sensitive test, because a next-but-one-item response is an immediate transposition error (i.e., an exchange between the correct item and the next item), easily the most common class of serial-order errors (cf. Henson, 1998). That is, this analysis may not have been sufficiently sensitive to reveal a real difference, due to the fact that other serial-order errors ("distant transposition errors") are relatively rare. However, we also examined whether next-but-one-item responses in the probe task were output more quickly in the alternatingvoices condition (with reaction times [RTs] recorded from the onset of the response display). This analysis did not suffer from the same shortcoming as the error analysis, because it involved comparing RTs for responding with the same item across conditions. The analysis of the RT data from the probe task (again using an even sampling of the probed positions) revealed that next-but-one-item responses were indeed output more quickly in the alternating-voice condition $(M=2.48 \mathrm{~s}, S E=0.13)$ than in the single-voice condition $(M=3.26 \mathrm{~s}, S E=0.27), t(1,17)=2.21, p<.05$ (note that this RT analysis involved the data from only the 18 participants from our sample of 26 who made at least one next-item response and at least one next-but-one-item response). We also found that the reverse was the case for outputting the correct response: When the correct response was output in the alternating-voice condition-which would, on our account, involve switching from one stream to the other-it was output more slowly $(M=2.75 \mathrm{~s}, S E=$ $0.12)$ than in the single-voice condition $(M=2.62 \mathrm{~s}, S E=$ $0.11)$. Although the latter difference on its own was not reliable $(p=.18)$, the interaction between voice condition 
and serial position [next item (i.e., the correct response) vs. next-but-one item] was indeed significant $F(1,17)=5.86$, $M S E=717,027.13, p<.03$. In sum, the next-but-one item (an error) was more quickly accessible, and the next item (the correct response) somewhat less quickly accessible, in the alternating-voice than in the single-voice condition.

No specific predictions were made about how the voice alternation effect might vary according to serial position. However, for the sake of completeness, Fig. 2a plots the data obtained from the probe task (again, with even sampling) according to list type across each probed item (i.e., Items 2-8). This indicates that the impairment from voice alternation is apparent throughout the curve, except for the last two positions. Whilst the reason for this absence of an effect at recency is not clear, we note that a similar pattern - the elimination at recency of an impairment found at prerecency - has been observed previously in studies using a similar, position-probe task (e.g., "What was the item in Position 3?"; Avons, Wright, \& Pammer, 1994; Elvevag, Fisher, \& Goldberg, 2002). A repeated measures ANOVA confirmed this impression of the data: There was a main effect of list type, $F(1,25)=33.265, M S E=.11, p<$ .001 , a main effect of serial position, $F(6,150)=3.181$, $M S E=.11, p<.01$, and an interaction between list type and serial position, $F(6,15)=3.44, M S E=.11, p<.01$.
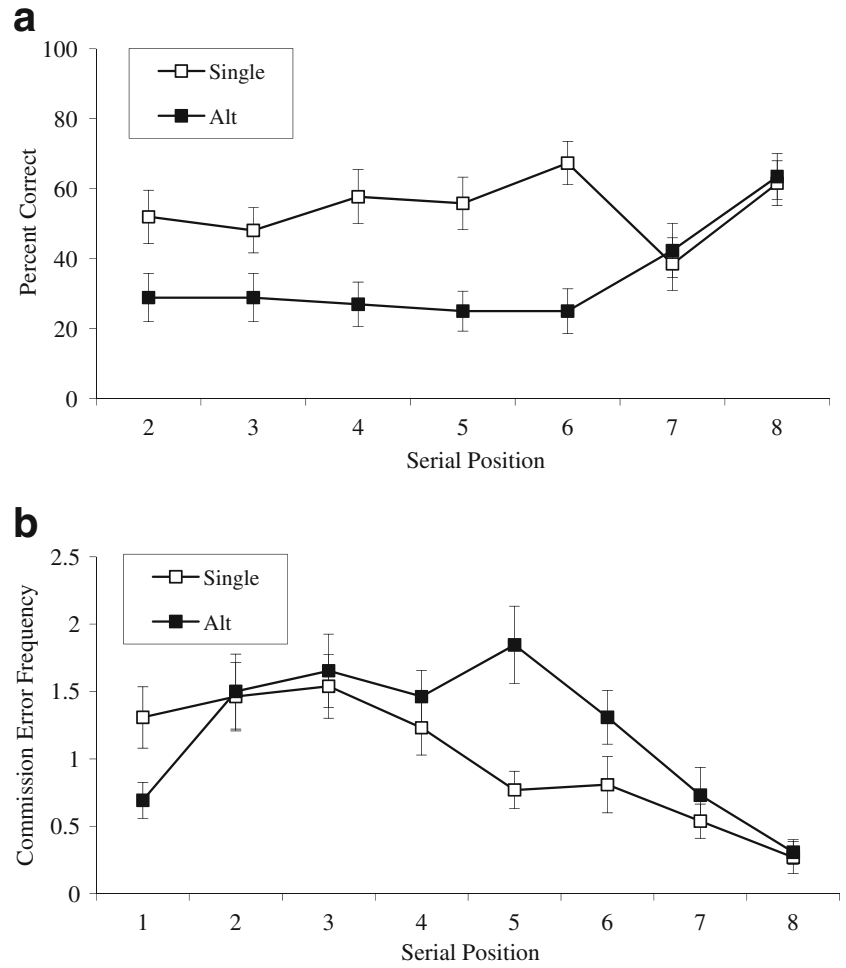

Fig. 2 a Mean percentages of items recalled in the probe task in Experiment 1, for each probed position according to list type. b Frequencies of errors of commission in the missing-item task in Experiment 1, for each serial position according to list type
For the missing-item task, it is not possible to generate a "standard" serial position function because the missing item of course does not have a serial position. However, a function can be generated that represents the frequency of errors of commission for each serial position-that is, errors are plotted according to the position in the presented list of the (erroneously) outputted item. Thus, given the list "37915264," outputting the item "1" (instead of the missing item, "8") would be scored as an error at Serial Position 4 (see Buschke \& Hinrichs, 1968; Hadley, Healy, \& Murdock, 1992). Figure 2b shows the errors of commission for the missing-item data from Experiment 1 according to list type. The function obtained in the single-voice condition suggests a small primacy effect and a stronger, extended recency effect, which replicates the function found in previous studies: Items are less likely to be selected incorrectly as the missing item if they were presented later in the list, and so, too, if they occur very early in the list (e.g., Buschke \& Hinrichs, 1968; Hadley et al., 1992). In general, talker variability appears to accentuate this tendency to incorrectly choose items from the middle of the list. It is not entirely clear why talker variability decreased the likelihood of incorrectly emitting the first-presented item as the missing item, unless this was simply a side effect of the exaggerated likelihood of incorrectly outputting middle as opposed to items at the list boundaries in this condition. A repeated measures ANOVA revealed a main effect of list type, $F(1,25)=$ 13.431, MSE = .30, $p<.001$, a main effect of serial position, $F(7,175)=8.248, M S E=1.24, p<.001$, and a significant interaction between list type and serial position, $F(7,175)=2.778, M S E=1.06, p<.01$.

The results of Experiment 1 are in line with the view that the compatibility between the products of perceptual organization and the true temporal order of the items places a key constraint on auditory-verbal serial STM performance: The impairment caused by voice alternation was appreciably and significantly larger in a task requiring serial-order retention (probed order recall) than in a task that was identical in terms of stimulus presentation and output demands but is not thought to rely on serial-order processing (missing-item task; e.g., Beaman \& Jones, 1997). A more detailed examination of the error and RT data from the probe task revealed further evidence consistent with the idea that the items were perceptually organized by voice in the alternating-voice condition: the next-but-one item was more accessible in the alternatingvoice condition (i.e., when that item would be the next within-stream item) than in the single-voice condition, both in the form of a numerical trend for greater frequency of outputting this (erroneous) item in the alternating-voice condition and, more compellingly in statistical terms, the significantly greater speed with which that error was 
committed, especially when considering the fact that the speed of outputting the correct response was numerically slower in that condition.

In sum, Experiment 1 suggests that a mismapping between perceptual organization and the actual serial order of the items had a marked impact on serial STM performance. Whereas in that experiment our approach was to examine the consequences of a mismatch between passive perceptual organization and serial-order requirements, in Experiment 2 we took the inverse approach: We examined a setting in which perceptual organization should map particularly well onto an effective serial rehearsal strategy and predicted, therefore, a facilitation rather than an impairment of probed order recall. A subsidiary issue addressed in this experiment was why missing-item recall still showed some degree of impairment from voice alternation.

\section{Experiment 2}

There is ample evidence that rehearsing a list in two temporally separated groups (e.g., 5714-8263) enhances serial recall (e.g., Ryan, 1969). In Experiment 2, we varied the talkers presenting an auditory-verbal list in such a way as to induce a perceptual organization that would map well onto this particularly effective two-discrete-groups strategy. Specifically, we again presented the eight items in two voices, but this time in two clusters (i.e., MMMMFFFF) rather than alternately. Such a separated-voices presentation format has already been shown to promote temporal grouping within the rehearsal plan and to facilitate serial recall (Frankish, 1985, 1989; Parmentier \& Maybery, 2008); thus, it is reasonable to expect probed order recall to also benefit from this compatible mapping between perceptual organization and an effective serial rehearsal strategy. The novel aspect of the experiment is that we again contrasted the effect of this form of talker variability on probed order recall with that on the missing-item task, our expectation being that missing-item recall should not be so influenced by whether or not the auditory perceptual organization maps well onto a two-discrete-group serial rehearsal strategy. There is already some evidence that lends credence to this expectation: Missing-item identification with visual presentation is not facilitated when such rehearsal grouping is promoted by presenting the list in two temporally defined groups (Klapp et al., 1983).

Whilst the key result from Experiment 1 was that probed order recall was impaired to a greater degree by voice alternation than was the missing-item task, Experiment 2 allowed us to also examine two accounts of why the missing-item task was disrupted at all. One possibility is that even though the missing-item task does not nominally require the retention of order, a serial rehearsal strategy might nevertheless be used to some extent (Norris, Baddeley, \& Page, 2004; see also Beaman \& Jones, 1998, for a discussion of the same issue in the context of free recall), thereby leaving it vulnerable to some influence of a mismatch between passive perceptual organization and actual item order. Another possibility, however, is that in the context of missing-item recall, it is not the voice alternation per se that impairs performance, but rather the fact that the list does not constitute one homogeneous set. That is, with two-voice lists, there is always more than one item missing from within each voice (or each subset; i.e., each voice only conveys four of the possible nine items that could be presented). It seems plausible that during the search at test for which item is relatively unfamiliar (cf. Buschke, 1963), the heterogeneity (in terms of input voice) creates an uncertainty as to whether a given candidate for the missing item was only missing from one of the voice sets or whether it was indeed missing from the entire list.

The use of two discrete voice groups (MMMMFFFF) in Experiment 2 should allow us to adjudicate between these two accounts: If missing-item recall was impaired in Experiment 1 due to a heterogeneity problem not because of a mismatch between perceived and actual order, we should again see an impairment (as compared to singlevoice presentation) in this task in the two-separated-voices condition, because the heterogeneous list problem would remain. This would be in contrast to the facilitation we would expect for probed order recall in the separated-voices condition. If, however, the impairment of missing-item identification from voice alternation was due to some use of serial rehearsal, both missing-item identification and probed order recall should show some enhancement in the separated-voices condition.

\section{Method}

Participants A group of 20 Cardiff University students, all reporting normal hearing and normal or corrected-to-normal vision, took part. None had participated in Experiment 1.

Apparatus and materials, design, and procedure All of these aspects of the methodology were identical to those of Experiment 1, except that the talker-variable condition on this occasion was a separated-voices condition, whereby one voice (female or male) conveyed the first four digits whilst the other voice conveyed the last four digits (which voice-female or male - conveyed the first four was counterbalanced).

Results and discussion

Figure 3 shows that for probed order recall, presenting two voices in separated groups, as opposed to alternately (Exp. 


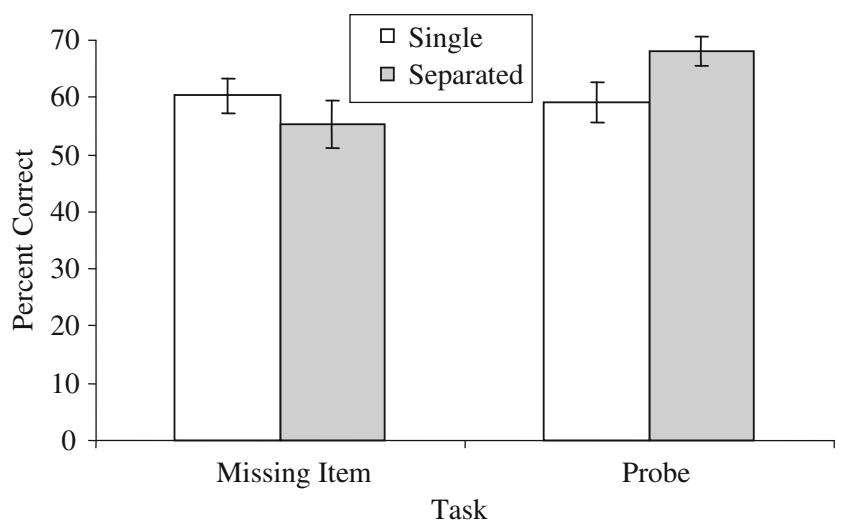

Fig. 3 Mean percentages of items correctly recalled in the single- and separated-voice conditions in the missing-item task and the probed order recall task in Experiment 2

1), reverses their impact: Separated-voice lists are better recalled than single-voice lists. In contrast, separated voices, like alternating voices in Experiment 1, impaired missing-item identification. Although there were no main effects of list type, $F(1,19)=1.66, M S E=.005, p>.05$, or task, $F(1,19)=1.60, M S E=.043, p>.05$, the critical interaction between list type and task was significant, $F(1$, 19) $=19.63, M S E=.005, p<.001$. Simple effects analyses showed that the single- and separated-voices conditions differed from one another in both the missing-item $(p<.05)$ and the probed order recall $(p<.001)$ tasks but, critically, in opposite directions. As in Experiment 1, the same pattern was found with even sampling in the probe task, with mean probed recall being $54.6 \%(S E=4.12$, single voice $)$ and $71.8 \%$ ( $S E=2.51$, separated voices).

Again, there was no difference between the tasks in the single-voice condition, $p=.82(p=.34$ using even sampling in the probe task). Further evidence in line with our contention that the key precondition for disruption of missing-item recall is the presence of two voices, not the particular manner in which they are arranged, was that the degree to which this task was impaired by separated voices was comparable statistically to the degree to which it was impaired by voice alternation in Experiment 1: Taking the data from only the missing-item task from both experiments, there was no interaction between list type (single vs. multiple voices) and experiment, $F<1$.

As in Experiment 1, for completeness we analyzed the probe data and also the frequency of errors of commission in the missing-item task for each serial position (panels A and B, respectively, in Fig. 4). The probe data indicate that the benefit of separated voices is apparent throughout most of the curve but seems to have been driven particularly by an advantage at the boundary marked by the change in voice after four items (i.e., Probed Position 5). This pattern may reflect the same "miniprimacy" effect found for subgroups within grouped serial-recall lists (see, e.g.,
Frankish, 1985). A repeated measures ANOVA revealed a main effect of list type, $F=37.09, M S E=.051, p<.001$, a main effect of serial position, $F=7.314, M S E=.12, p<$ .001 , and a significant interaction between these two factors, $F=2.44, M S E=.091, p<.05$.

The errors-of-commission analysis for the missing-item task (Fig. 4b) reveals a general pattern similar to that found in Experiment 1: Following a short-lived primacy effect, there is an extended declination in the likelihood of incorrectly selecting an item as the missing item as a function of list position (cf. Buschke \& Hinrichs, 1968). Moreover, the impact of talker variability on errors of commission again tends to be one of accentuating the likelihood of erroneously selecting midlist items. There were significant main effects of list type, $F(1,19)=6.40$, $M S E=.26, p<.05$, and of serial position, $F(7,133)=$ 6.979, $M S E=1.26, p<.001$. Unlike in Experiment 1, the interaction of these two factors was not significant; however, the absence of a three-way interaction between experiment, list type, and serial position, $F(7,308)=1.5$, $M S E=.99, p>.05$, tends to militate against placing too much theoretical weight on this discrepancy.

The facilitative effect of separated voices on probed order recall is consistent with numerous studies showing the benefits of grouping on serial recall (e.g., Frankish,

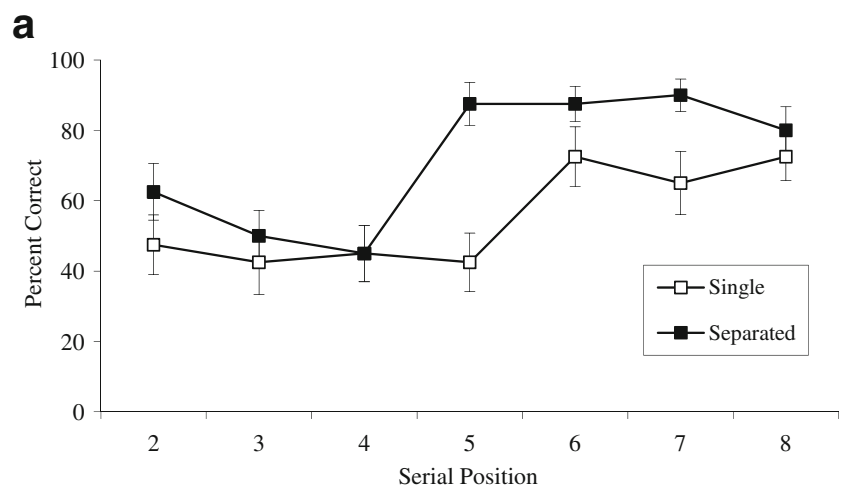

b

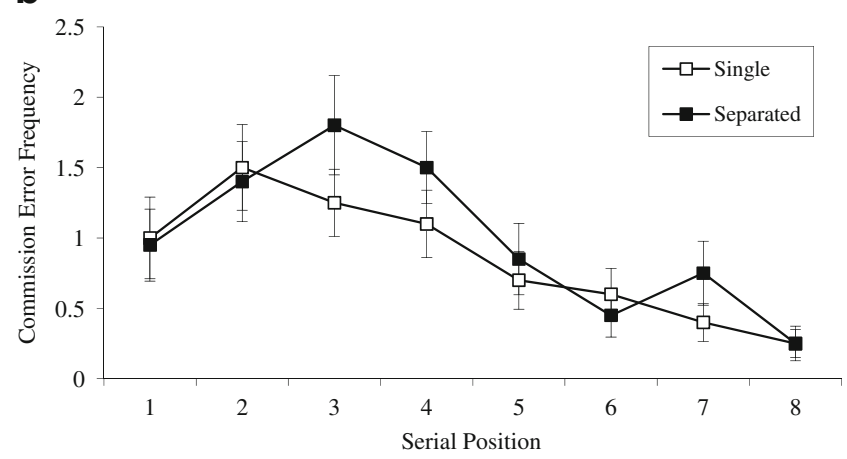

Fig. 4 a Mean percentages of items recalled in the probe task in Experiment 2, for each probed position according to list type. b Frequencies of errors of commission in the missing-item task in Experiment 2, for each serial position according to list type 
1985, 1989; Parmentier \& Maybery, 2008; Ryan, 1969). We suggest that this results from, on this occasion, a compatibility in the mapping between perceptual organization and an effective serial rehearsal strategy. Moreover, the fact that missing-item identification is impaired (and to a comparable extent) by the presence of two voices, regardless of how talker variability is implemented (i.e., alternating or separated), suggests that the underlying mechanism for the impairment found in this task due to talker variability (Exp. 1 ) is qualitatively distinct from that found in order recall tasks.

The novel finding that missing-item identification is also impaired by talker variability to some extent, regardless of the way in which the different voices are arranged, seems consistent with the view that missing-item identification is based on an item (un)familiarity judgment (Buschke, 1963; Buschke \& Hinrichs, 1968): It is reasonable to suppose that such a judgment would be more difficult when there is an ambiguity as to whether an item is unfamiliar in just one of two input voices or is unfamiliar regardless of voice (i.e., is missing from the entire list). If this interpretation is correct, a further, broader implication of these results is that the physical (e.g., pitch, timbre) and/ or indexical (e.g., accent, gender) characteristics of the spoken items seems to be retained, in line with episodic, as opposed to abstractionist, views of memory (Goldinger, 1998; Port, 2010). If verbal items were represented purely in terms of idealized, abstract phonological codes, it is difficult to see why performance would be influenced by such precategorical attributes. At the very least, it seems that the manipulation of talker variability may provide a valuable tool to investigate in more detail how the missingitem task is performed.

\section{General discussion}

To summarize, Experiment 1 showed that voice alternation impairs an STM task calling for serial-order retention (probed order recall) to a much greater degree than a task that calls for item but not order retention (missing-item task). This is in line with the view that the perceptual organization of alternating voices into voicebased streams yields order cues that are incompatible with the requirement to recall the order of items as presented (Hughes et al., 2009); when the task does not require order recall, those order cues become immaterial. Further analysis of errors and RT data from the probe task in Experiment 1 provided some novel evidence for byvoice organization: An error type in which the next-butone item was output instead of the next item was made more quickly in the alternating-voice condition - that is, when the next-but-one item would be the next item within the same voice-based stream that conveyed the probe item. In a complementary fashion, Experiment 2 showed that with separated-voices presentation-in which perceptual organization maps well onto an effective serial rehearsal strategy-probed order recall, but not missing-item recall, is facilitated. Indeed, missing-item recall was again impaired by talker variability in this experiment, suggesting that the impairment to this task by voice alternation in Experiment 1 was not related to stream segregation, but was more likely due to an ambiguity as to whether the critical item was missing from the entire list or from only one of the (voice-defined) sub-sets. Whilst further work will be required to evaluate this account of the effect of talker variability on missing-item recall, the focus for the remainder of our discussion will be on considering its effect on order-based tasks in the context of three broad approaches to serial STM.

\section{A perceptual-gestural account}

The perceptual-organization-based account of the effects of talker variability on order recall can be set within the broader view that serial STM performance reflects constraints on general-purpose perceptual and motor sequenceplanning processes and the mapping between these processes. This perceptual-gestural view (Hughes et al., 2009; Jones, Hughes, \& Macken, 2006; 2007; Jones, Macken, \& Nicholls, 2004; Macken \& Jones, 2003) supposes that the assembly of a coarticulated motor analogue (articulatory in the case of verbal material) of the list is the primary vehicle by which order cues are grafted onto the to-be-remembered material to support its short-term retention and reproduction. From this standpoint, serial rehearsal in STM tasks is akin to a covert simulation (or forward model; cf. Grush, 2004) of those bodily movements that are best placed to mimic the presented material. Thus, vocal-articulatory movements will tend to be deployed with verbal materials because the participant will typically already be endowed with the vocal-articulatory skills for reproducing written or heard language. Similarly, voluntary eye movements may be the main vehicle by which visuospatial sequences are retained (e.g., Tremblay, Saint-Aubin, \& Jalbert, 2006). A motor analogue is needed to serve as "carrier" in this way because order cues are impoverished in the serial verbal STM list, due to the absence, by design, of the syntactical and semantic structure that usually provides such cues in everyday verbal sequences or, likewise, the absence of recognizable shapes (e.g., a square) in visuospatial serial STM tasks. Thus, for verbal lists, paralinguistic aspects of the skill of speech planning such as coarticulation (e.g., Farnetani \& Recasens, 1999; Woodward, Macken, \& Jones, 2008) and prosody (e.g., Reeves, Schmauder, \& Morris, 2000) are co-opted in an opportunistic fashion so as to 
embellish the presented material with cues to the order of successive events. ${ }^{1}$

Set within this framework, the disruptive effect of voice alternation on probed order recall (Exp. 1) and serial recall (Hughes et al., 2009) may be understood in terms of a difficulty in mapping the products of an involuntary, perceptual sequencing process (e.g., Bregman, 1990) onto a controlled, motor sequencing one (e.g., Rosenbaum, 1991). In line with forward models of motor control, the perceptual-gestural account assumes that the uploading of the sequence of elements comprising the motor program proceeds in real time and at a speed that is constrained by the effector movements (those of the vocal tract, in this case) being simulated, as has been shown in other motor domains (e.g., Decety, Jeannerod, \& Prablanc, 1989; Reed, 2002). It follows that when the elements of the program are dictated by external input (as opposed to elements from long-term memory) - as is the case in serial STM tasksthere are likely to be settings in which the structure or organization of the input is not conducive to this timely "pick-up" of items in the correct, to-be-coarticulated sequence. We argue that voice alternation is one such setting, where fast, automatic streaming processes yield sequential information that is at odds with, or at least is ambiguous in relation to, the true temporal order of the items. Conversely, a perceptual organization that promotes the imposition of prosodic boundaries within the motor plan (Exp. 2) facilitates order recall by providing strong order

\footnotetext{
${ }^{1}$ At first glance, our emphasis on gestural processes in serial STM performance would seem to run into difficulties in relation to some of the neuropsychological literature on serial STM. Patients with dysarthria - a deficit of speech production - exhibit a phonological similarity effect (as well as a word-length effect), both of which are thought, according to the perceptual-gestural account, to primarily reflect the use of motor processes in serial STM (e.g., Jones et al., 2004). However, it is important to distinguish between speech (or more generally, gestural) planning and speech/gesture production, and the former is what plays a key role in the perceptual-gestural account (indeed, no sequence production is required in the present probe-order task, notwithstanding the sequencing of the motor act involved in pressing a single key). Consistent with the perceptual-gestural account, patients with a deficit of speech planning (apraxia of speech), however, do not show a phonological similarity effect for visually presented lists or a word-length effect (Waters, Rochon, \& Caplan, 1992; the residual phonological similarity effect for auditory lists may be attributable to intact perceptual organization processes - see Jones et al., 2004-although this remains an empirical question at present). Thus, Waters et al. concluded that such "results are in contrast to those of other studies in which patients with dysarthria, a lower-level speech output impairment, have been tested and they suggest that the speechbased process involved in planning the articulation of speech sounds, rather than in production, plays a role in rehearsal mechanisms" ( $p$. 54). As Waters et al. pointed out in their discussion of Bishop and Robson's (1989) study, it also cannot be ruled out that congenitally anarthric children have some capacity to develop speech-planning abilities, hence their showing of a phonological similarity effect and the word-length effect.
}

cues for items at the group boundaries, thereby minimizing erroneous exchanges of elements of the speech plan that traverse those boundaries (see, e.g., Dell, 1984; Henson, 1998; Parmentier \& Maybery, 2008).

Whilst the present results are broadly consistent with a perceptual-gestural approach, it is important to acknowledge that the experiments were not designed specifically to rule out other possible accounts of talker variability effects in serial STM. However, coupled with the findings of Hughes et al. (2009), there seems now to be a sufficient body of data to begin adjudicating between different accounts. We now therefore consider in some detail two other theoretical approaches, one based on the concept of a distinct phonological short-term store (see, e.g., Baddeley, 1986, 2007) and another on the notion that a limited-capacity focus of attention plays a central role in STM (e.g., Cowan, 1995, 2001).

\section{Phonological store-based accounts}

Some previous accounts of talker variability effects in serial STM have appealed to item-level processes (Goldinger et al., 1991; Martin et al., 1989) and are couched in terms of the phonological loop component of the working memory model (Baddeley \& Hitch, 1974; for more recent accounts, see Baddeley, 2007; Baddeley \& Larsen, 2007) rather than perceptual-sequence organization processes, as emphasized here. For example, Martin et al. (1989) suggested that with a multiple-voice list there may be a cost associated with discarding the task-irrelevant variation in the voice (e.g., pitch, accent) conveying each successive item as part of a speech normalization process (cf. Pisoni, 1997). Conversely, Goldinger et al. (1991) suggested that the cost might be due to an obligatory incorporation of the variation in voice. Specifically, these accounts suggest that the delay in processing each item during encoding or/and rehearsal decreases the probability of the items being transferred to a more durable long-term store from which they need to be retrieved, due presumably to the delay allowing more opportunity for the items to decay and be lost from the passive phonological short-term store (cf. Baddeley, Thomson, \& Buchanan's, 1975, account of the word-length effect).

From one standpoint, it may not be appropriate to expect these particular phonological-store-based accounts (Goldinger et al., 1991; Martin et al., 1989) to apply to our present and recent findings (cf. Hughes et al., 2009). These accounts are based on studies using a relatively long list of words (10 items) and in which the words were different for each trial. In contrast, we used a more standard serial STM task involving a relatively short (eight-item) list comprising items from a small restricted set (present study; Hughes et al., 2009; see also Greene, 1991). An explanation based on failure of the transfer of items from short- to long-term stores due to itemprocessing costs (Goldinger et al., 1991; Martin et al., 1989) 
might therefore apply with long lists and where there is a heavy burden on item (as opposed to order) recall, but the applicability of this approach to studies using shorter lists in which order, not item, recall dominates is less apparent. From another standpoint, however, an item-processing cost approach to the standard paradigm could indeed be viewed as reasonable, in light of the fact that, in the phonological loop model, a similar explanation is offered for the word-length effect (which is typically based on studies with short, closedset lists; e.g., Baddeley et al., 1975). This effect has also been explained in terms of an item-processing cost on this approach: Having to encode and rehearse long rather than short words allows for greater opportunity for decay, and hence for loss of items from the phonological store (Baddeley et al., 1975), as is supposed also for multiple- as compared to single-voice items (e.g., Goldinger et al., 1991).

Assuming that an item-processing cost approach can, in principle, be applied more broadly, how well does such an approach fare? A distinct strength of the item-processing cost approach - particularly the voice incorporation account (Goldinger et al., 1991) - is that it provides an explanation for the finding that recall of lists in which each item is presented in a unique voice is better than recall of singlevoice lists when the rate of presentation is slowed down markedly (to one item per $4 \mathrm{~s}$; Goldinger et al., 1991). According to Goldinger et al., the delay associated with retaining the idiosyncratic voice information exerts a cost at relatively fast rates, but that same information can serve as an additional cue, at retrieval, to each item's identity if the time pressure is lifted through use of a very slow presentation rate, and hence can benefit recall.

However, whereas item-processing costs (and benefits at slow presentation rates) may play a role when there is a heavy burden on recalling item identity, such as was the case in the procedure of Goldinger et al. (1991) and Martin et al. (1989), such an approach does not fare so well in relation to the talker variability effects found in more standard serial STM tasks (present study; Greene, 1991; Hughes et al., 2009). The studies of Goldinger et al. and Martin et al., as with early studies using free recall (Watkins \& Watkins, 1980), presented each item in a unique voice, whereas the studies in which we have found an impairment to serial STM due to multiple-voice presentation (Hughes et al., 2009; present Exp. 1; see also Greene, 1991) have involved just two voices presented in a regular, alternating pattern. As noted by Greene (1991), item-processing costs "should be minimized by reducing the number of voices and making their occurrence highly predictable" (p.76). Furthermore, there are data that directly contradict this prediction of the item-processing cost accounts: In the context of serial recall of an eight-item list, presenting the items in two alternating voices is more, not less, damaging than presenting the items in four voices (Hughes et al.,
2009, Exp. 3). The latter finding can be readily explained in terms of sequence-level perceptual organization processes, however: With a four-voice list, the tendency for nonadjacent items to cohere perceptually would be diminished as compared to an alternating-voice list, because in a fourvoice list the number of nonadjacent pairs of items spoken in the same voice is necessarily smaller. It is also not clear, if the negative effect of talker variability is due to an itemprocessing cost, why tasks that emphasize retention of item over order information - such as the missing-item task used here-are less susceptible to that effect (present experiments). Another challenge for this approach is to explain the influence of a lead-in on the impact of voice alternation on serial recall. As noted earlier, preexposure to the voices to be heard in the to-be-remembered list increases the magnitude of the voice alternation effect in serial recall (Hughes et al., 2009, Exps. 1 and 2). It might be expected that preexposure to the voices, and particularly to the pattern of voice changes, should, if anything, facilitate a voice normalization or incorporation process, contrary to what the data indicate.

Talker variability effects in serial STM may, however, be better accommodated by a phonological-store-based approach that acknowledges a more central role for auditory streaming in serial STM. Whilst acoustic-sensory processing has, historically, been viewed as "peripheral to the working memory system" (Baddeley, 1986, p.95), one contemporary computational instantiation of the phonological loop-the primacy model - has indeed already appealed to such processes in relation to the effects of presentation modality (Page \& Norris, 1998) and irrelevant sound (Page \& Norris, 2003). According to the primacy model, item order is represented by a primacy gradient of item activation strengths, and thus it might be argued that voice alternation modulates the representation of order in the phonological store by setting up two interleaving primacy gradients, one representing each voice-based stream. The cost to strict serial-order recall might follow from having to switch between the two gradients at retrieval. Another possibility is that perceptual organization by voice results in two competing sets of item-position cues (e.g., Burgess \& Hitch, 1999), one based on true temporal position and one based on within-voice position, again thereby causing interference during retrieval from a phonological store rather than impairing the pick-up of items into a motor plan.

Two strands of evidence provide an initial challenge to the store-based accounts, however. First, given that auditory input is assumed to gain obligatory access to the passive phonological store (see, e.g., Baddeley, 2007), the effects of talker variability should still be evident when access to articulatory processes are impeded by articulatory suppression (as with the phonological similarity effect; cf. Baddeley, Lewis, \& Vallar, 1984). However, some data 
indicate that articulatory suppression does eliminate the damaging effect of talker variability on order recall (Chamberland, Chamard, Hodgetts, Jones, \& Tremblay, 2009). A second challenge relates to how talker variability might be expected to interact with phonological similarity. If talker variability is assumed to affect item order cues within the phonological store, this variable should not modulate another effect - the phonological similarity effect (the difficulty of recalling, e.g., "B, C, D, E,..." as compared to "K, F, R, Q,..."; see, e.g., Conrad, 1964)that is assumed on these models to be located at a later and independent item-retrieval stage of recall (Burgess \& Hitch, 1999; Page \& Norris, 1998). However, talker variability does indeed markedly diminish the phonological similarity effect (Hughes et al., 2009, Exp. 4). Note, however, that both of the foregoing lines of evidence are consistent with our proposal that the talker variability effect (and so, too, the phonological similarity effect in the absence of articulatory suppression; see Jones et al., 2004) is located ultimately in the articulatory sequence-planning process.

\section{Attentional cost account}

Another alternative approach to talker variability effects may be couched in terms of Cowan's $(1995,2001)$ embeddedprocesses model of working memory. This model posits that performance of STM tasks relies in part on the ability to keep items active in a limited-capacity "focus of attention," a subcomponent embedded within a larger set of temporarily active and highly accessible items from within permanent (or long-term) memory. According to this model, it might be suggested that voice alternation impairs probed order recall (Exp. 1) as well as serial recall (Greene, 1991; Hughes et al., 2009) because such a condition incurs an attentional cost: For example, rather than invoking perceptual streaming processes, it might be supposed that there is a delay involved in switching attention between voices (or frequency bands), as seems to be the case when items alternate between ears (e. g., Cherry \& Taylor, 1954), which may in turn lead to a greater likelihood of decay of already-encoded items from the limited focus of attention. However, the same data that pose difficulties for the voice normalization (Martin et al., 1989) and voice incorporation (Goldinger et al., 1991) accounts are also problematic for this type of attentional account: For example, one might expect preexposure to the pattern of voice changes to, if anything, facilitate the attention-switching process and lead to less impairment, whereas preexposure to the pattern in fact increases the effect (Hughes et al., 2009). Moreover, having four different frequency bands to switch between should, presumably, impede recall more than a setting in which attention needs to switch between just two voices changing in a highly predictable fashion. Thus, the fact that two voices alternating are more damaging than four voices (Hughes et al., 2009, Exp. 3) is also not readily accommodated within an attentional cost account.

However, it could be argued that whether or not the impairment due to voice alternation is appropriately characterized as an attentional cost depends on what is meant by the rather nebulous concept of attention. On Cowan's model, attention seems to be construed as a distinct resource (cf. Kahneman, 1973), such that items in STM are lost if this resource is withdrawn for a sufficient time. However, within a perceptual-gestural mismapping account (Hughes et al., 2009), the impairment could also be described as an attentional cost if selective attention is construed instead as the process of coupling object-based representations (e.g., "streams" in the auditory domain) to motor skills (cf. the selection-for-action framework; Allport, 1993; Houghton \& Tipper, 1996; Hughes \& Jones, 2005; Neumann, 1987). In this view, the impairment may indeed be appropriately characterized as being due to having to switch attention between two objects generated by preattentive streaming processes. Moreover, the difficulty caused by the preattentive creation of two objects on this account is in uploading the items in the correct order into a motor sequence plan, not the decay of item information. Again, the fact that order rather than item memory is particularly vulnerable to voice alternation (Exp. 1) seems to support this view.

\section{Conclusions}

On the basis of the present and recent related findings (Hughes et al., 2009), we argue that talker variability effects in order recall tasks highlight a key role for obligatory auditory perceptual organization processes in serial STM performance (see also Frankish, 1985, 1989; Jones et al., 2006; Jones et al., 2004; Nicholls \& Jones, 2002). Moreover, the available evidence to date suggests that the ultimate locus of the effects is in the mapping between an ordered representation derived from that perceptual organization and a deliberately assembled sequence plan: When the match between the two modes of organization is relatively poor, order recall is impaired (present Exp. 1; Greene, 1991; Hughes et al., 2009), whereas when the match is good, order recall is facilitated (present Exp. 2). One important avenue for further investigation, however, will be to determine the balance between the contributions to talker variability effects of the sequence-level processes emphasized here and other, item-level mechanisms (e.g., voice normalization or incorporation processes; see, e.g., Goldinger et al., 1991) as a function of the degree to which an order recall task also demands recall of item identity and how the balance of these contributions may, in turn, be modulated by the rate at which items are presented (see Goldinger et al., 1991; Nygaard et al., 1995). 
Author note This research was supported by the United Kingdom's Economic and Social Research Council in the form of a grant awarded to D.M.J., R.W.H., and Bill Macken (RES-062-23-0011). Thanks are due Bill Macken and Phillip Beaman for useful discussions about the work presented here.

\section{References}

Allport, D. A. (1993). Attention and control: Have we been asking the wrong questions? A critical review of 25 years. In D. E. Meyer \& S. Kornblum (Eds.), Attention and performance XIV: Synergies in experimental psychology, artificial intelligence, and cognitive neuroscience (pp. 183-218). Cambridge, MA: MIT Press.

Anstis, S., \& Saida, S. (1985). Adaptation to auditory streaming of frequency modulated tones. Journal of Experimental Psychology. Human Perception and Performance, 11, 257-271.

Avons, S. E., Wright, K. L., \& Pammer, K. (1994). The word-length effect in probed and serial recall. The Quarterly Journal of Experimental Psychology, 47A, 207-231. doi:10.1080/ 14640749408401151

Baddeley, A. (1986). Working memory. Oxford: Oxford University Press, Clarendon Press.

Baddeley, A. (2007). Working memory, thought, and action. Oxford: Oxford University Press.

Baddeley, A. D., \& Hitch, G. J. (1974). Working memory. In G. H. Bower (Ed.), The psychology of learning and motivation (Vol. 8, pp. 47-89). New York: Academic Press.

Baddeley, A. D., \& Larsen, J. D. (2007). The phonological loop unmasked? A comment on the evidence for a "perceptualgestural" alternative. The Quarterly Journal of Experimental Psychology, 60, 497-504. doi:10.1080/17470210601147572

Baddeley, A. D., Thomson, N., \& Buchanan, M. (1975). Word length and the structure of short-term memory. Journal of Verbal Learning and Verbal Behavior, 14, 575-589. doi:10.1016/ S0022-5371(75)80045-4

Baddeley, A., Lewis, V., \& Vallar, G. (1984). Exploring the articulatory loop. The Quarterly Journal of Experimental Psychology, 36A, 233-252. doi:10.1080/14640748408402157

Baddeley, A., Gathercole, S., \& Papagno, C. (1998). The phonological loop as a language learning device. Psychological Review, 105, 158-173. doi:10.1037/0033-295X.105.1.158

Beaman, C. P., \& Jones, D. M. (1997). The role of serial order in the irrelevant speech effect: tests of the changing state hypothesis. Journal of Experimental Psychology. Learning, Memory, and Cognition, 23, 459-471.

Beaman, C. P., \& Jones, D. M. (1998). Irrelevant sound disrupts order information in free recall as in serial recall. The Quarterly Journal of Experimental Psychology, 51A, 615-636. doi:10.1080/027249898391558

Bhatarah, P., Ward, G., \& Tan, L. (2008). Examining the relationship between free recall and immediate serial recall: the serial nature of recall and the effect of test expectancy. Memory \& Cognition, 36, 20-34. doi:10.3758/MC.36.1.20

Bishop, D. V. M., \& Robson, J. (1989). Unimpaired short-term memory and rhyme judgement in congenitally speechless individuals: implications for the notion of "articulatory coding. The Quarterly Journal of Experimental Psychology, 41A, 123140. doi:10.1080/14640748908402356

Bregman, A. S. (1990). Auditory scene analysis: The perceptual organisation of sound. Cambridge, MA: MIT Press.

Bregman, A. S., \& Campbell, J. (1971). Primary auditory stream segregation and perception of order in rapid sequences of tones. Journal of Experimental Psychology, 89, 244-249.
Bregman, A. S., \& Rudnicky, A. I. (1975). Auditory segregation: stream or streams? Journal of Experimental Psychology. Human Perception and Performance, 1, 263-267.

Burgess, N., \& Hitch, G. (1999). Memory for serial order: a network model of the phonological loop and its timing. Psychological Review, 106, 551-581.

Buschke, H. (1963). Relative retention in immediate memory determined by the missing scan method. Nature, 200, 1129-1130.

Buschke, H., \& Hinrichs, J. V. (1968). Relative vulnerability of item information in short-term storage for the missing scan. Journal of Verbal Learning and Verbal Behavior, 7, 1043-1048. doi:10.1016/S0022-5371(68)80065-9

Chamberland, C., Chamard, E., Hodgetts, H. M., Jones, D. M., \& Tremblay, S. (2009, November). Serial memory for lists of spoken items: An examination of talker location and voice changes. Paper presented at the 8th Annual Auditory Perception, Cognition, and Action Meeting, Boston, MA.

Cherry, E. C., \& Taylor, W. K. (1954). Some further experiments upon the recognition of speech, with one and with two ears. The Journal of the Acoustical Society of America, 26, 554-559.

Conrad, R. (1964). Acoustic confusions in immediate memory. British Journal of Psychology, 55, 75-84.

Cowan, N. (1995). Attention and memory: An integrated framework. Oxford: Oxford University Press.

Cowan, N. (2001). The magical number 4 in short-term memory: a reconsideration of mental storage capacity. The Behavioral and Brain Sciences, 24, 87-185.

Decety, J., Jeannerod, M., \& Prablanc, C. (1989). The timing of mentally represented actions. Behavioral Brain Research, 34, 35-42.

Dell, G. S. (1984). Representation of serial order in speech: evidence from the repeated phoneme effect in speech errors. Journal of Experimental Psychology. Learning, Memory, and Cognition, 10, 222-233.

Elvevag, B., Fisher, J. E., \& Goldberg, T. E. (2002). Probed recall for serial order deficits in short-term memory in schizophrenic patients. Schizophrenia Research, 59, 127-135.

Farnetani, E., \& Recasens, D. (1999). Coarticulation models in recent speech production theories. In W. J. Harcastle \& N. Hewlett (Eds.), Coarticulation: Theory, data and techniques. Cambridge: Cambridge University Press.

Frankish, C. (1985). Modality-specific grouping effects in short-term memory. Journal of Memory and Language, 24, 200-209. doi:10.1016/0749-596X(85)90024-5

Frankish, C. (1989). Perceptual organization and precategorical acoustic storage. Journal of Experimental Psychology. Learning, Memory, and Cognition, 15, 469-479. doi:10.1037/02787393.15.3.469

Goldinger, S. D. (1998). Echoes of echoes? An episodic theory of lexical access. Psychological Review, 105, 251-279.

Goldinger, S. D., Pisoni, D. B., \& Logan, J. S. (1991). On the nature of talker variability effects in recall of spoken word lists. Journal of Experimental Psychology. Learning, Memory, and Cognition, $17,152-162$.

Greene, R. L. (1991). Serial recall of two-voice lists: implications for theories of auditory recency and suffix effects. Memory \& Cognition, 19, 72-78.

Grush, R. (2004). The emulation theory of representation: motor control, imagery, and perception. The Behavioral and Brain Sciences, 27, 377-442.

Hadley, J. A., Healy, A. F., \& Murdock, B. B. (1992). Output and retrieval interference in the missing-number task. Memory \& Cognition, 20, 69-82.

Henson, R. N. A. (1998). Short-term memory for serial order: the start-end model. Cognitive Psychology, 36, 73-137.

Houghton, G., \& Tipper, S. P. (1996). Inhibitory mechanisms of neural and cognitive control: applications to selective attention and sequential action. Brain and Cognition, 30, 20-43. 
Hughes, R. W., \& Jones, D. M. (2005). The impact of order incongruence between a task-irrelevant auditory sequence and a task-relevant visual sequence. Journal of Experimental Psychology. Human Perception and Performance, 31, 316327.

Hughes, R. W., Vachon, F., \& Jones, D. M. (2007). Disruption of short-term memory by changing and deviant sounds: support for a duplex-mechanism account of auditory distraction. Journal of Experimental Psychology. Learning, Memory, and Cognition, 33, $1050-1061$.

Hughes, R. W., Marsh, J. E., \& Jones, D. M. (2009). Perceptualgestural (mis)mapping in serial short-term memory: the impact of talker variability. Journal of Experimental Psychology. Learning, Memory, and Cognition, 35, 1411-1425.

Humphreys, M. S., \& Schwartz, R. M. (1971). Within category generalization in a missing scan paradigm. Journal of Verbal Learning and Verbal Behavior, 10, 694-701.

Jones, D. M., \& Macken, W. J. (1993). Irrelevant tones produce an irrelevant speech effect: implications for phonological coding in working memory. Journal of Experimental Psychology. Learning, Memory, and Cognition, 19, 369-381.

Jones, D. M., Macken, W. J., \& Nicholls, A. P. (2004). The phonological store of working memory: is it phonological, and is it a store? Journal of Experimental Psychology. Learning, Memory, and Cognition, 30, 656-674.

Jones, D. M., Hughes, R. W., \& Macken, W. J. (2006). Perceptual organization masquerading as phonological storage: further support for a perceptual-gestural view of short-term memory. Journal of Memory and Language, 54, 265-281.

Jones, D. M., Hughes, R. W., \& Macken, W. J. (2007). The phonological store abandoned. The Quarterly Journal of Experimental Psychology, 60, 505-511. doi:10.1080/17470210601147598

Kahneman, D. (1973). Attention and effort. Englewood Cliffs, NJ: Prentice Hall.

Klapp, S. T., Marshburn, E. A., \& Lester, P. T. (1983). Short-term memory does not involve working memory of information processing: the demise of a common assumption. Journal of Experimental Psychology. General, 112, 240-264.

Macken, W. J., \& Jones, D. M. (1995). Functional characteristics of the inner voice and the inner ear: single or double agency? Journal of Experimental Psychology. Learning, Memory, and Cognition, 21, 436-448.

Macken, W. J., \& Jones, D. M. (2003). Reification of phonological storage. The Quarterly Journal of Experimental Psychology, 56A, 1279-1288. doi:10.1080/02724980245000052

Macken, W. J., Tremblay, S., Houghton, R., Nicholls, A. P., \& Jones, D. M. (2003). Does auditory streaming require attention? Evidence from attentional selectivity in short-term memory. Journal of Experimental Psychology. Human Perception and Performance, 29, 43-51.

Martin, C. S., Mullennix, J. W., Pisoni, D. B., \& Summers, W. V. (1987). Effects of talker variability on recall of spoken word lists. Journal of Experimental Psychology. Learning, Memory, and Cognition, 15, 676-684.

Miller, G. A., \& Heise, G. A. (1950). The trill threshold. The Journal of the Acoustical Society of America, 22, 637-638.

Murdock, B. B., Jr. (1968). Serial order effects in short-term memory. Journal of Experimental Psychology, 76(4, Pt.2), 1-15. doi: $10.1037 / \mathrm{h} 0025694$
Neumann, O. (1987). Beyond capacity: A functional view of attention. In H. Heuer \& A. F. Sanders (Eds.), Perspectives on perception and action (pp. 361-394). Hillsdale: Erlbaum.

Nicholls, A. P., \& Jones, D. M. (2002). Capturing the suffix: cognitive streaming in immediate serial recall. Journal of Experimental Psychology. Learning, Memory, and Cognition, 28, 12-28.

Norris, D., Baddeley, A. D., \& Page, M. P. A. (2004). Retroactive effects of irrelevant speech on serial recall from short-term memory. Journal of Experimental Psychology. Learning, Memory, and Cognition, 30, 1093-1105.

Nygaard, L. C., Sommers, M. S., \& Pisoni, D. B. (1995). Effects of stimulus variability on perception and representation of spoken words in memory. Perception \& Psychophysics, 57, 989-1001.

Page, M. P. A., \& Norris, D. (1998). The primacy model: a new model of immediate serial recall. Psychological Review, 105, 761-781. doi:10.1037/0033-295X.105.4.761-781

Page, M. P. A., \& Norris, D. G. (2003). The irrelevant sound effect: what needs modelling, and a tentative model. The Quarterly Journal of Experimental Psychology, 56A, 1289-1300. doi:10.1080/ 02724980343000233

Parmentier, F. B. R., \& Maybery, M. T. (2008). Equivalent effects of grouping by time, voice, and location on response timing in verbal serial memory. Journal of Experimental Psychology. Learning, Memory, and Cognition, 34, 1349-1355.

Pisoni, D. B. (1997). Some thoughts on "normalization" in speech perception. In K. A. Johnson \& J. Mullennix (Eds.), Talker variability in speech processing (pp. 9-32). New York: Academic Press.

Port, R. F. (2010). Rich memory and distributed phonology. Language Sciences, 32, 43-55.

Reed, C. (2002). Chronometric comparisons of imagery to action: visualizing versus physically performing springboard dives. Memory \& Cognition, 30, 1169-1178.

Reeves, C., Schmauder, A. R., \& Morris, R. K. (2000). Stress grouping improves performance on an immediate serial list recall task. Journal of Experimental Psychology, 26, 1638-1654.

Rogers, W. L., \& Bregman, A. S. (1993). An experimental evaluation of three theories of auditory stream segregation. Perception \& Psychophysics, 53, 179-189.

Rosenbaum, D. A. (1991). Human motor control. San Diego, CA: Academic Press.

Ryan, J. (1969). Grouping and short-term memory: different means and patterns of grouping. The Quarterly Journal of Experimental Psychology, 21, 137-147. doi:10.1080/14640746908400206

Tremblay, S., Saint-Aubin, J., \& Jalbert, A. (2006). Rehearsal in serial memory for visual-spatial information: evidence from eye movements. Psychonomic Bulletin \& Review, 13, 452-457.

Van Noorden, L. P. A. (1975). Temporal coherence in the perception of tone sequences. Technische Hogeschool Eindhoven, Eindhoven, The Netherlands: Unpublished doctoral dissertation.

Waters, G. S., Rochon, E., \& Caplan, D. (1992). The role of high-level speech planning in rehearsal: evidence from patients with apraxia of speech. Journal of Memory and Language, 31, 54-73.

Watkins, O. C., \& Watkins, M. J. (1980). Echoic memory and voice quality: recency recall is not enhanced by varying presentation voice. Memory \& Cognition, 8, 26-30.

Woodward, A. J., Macken, W. J., \& Jones, D. M. (2008). Linguistic familiarity in short-term memory: a role for (co-)articulatory fluency? Journal of Memory and Language, 58, 48-65. doi:10.1016/j.jml.2007.07.002 